\title{
Epidemiology and Recurrence of Nursemaid's Elbow in Children with Ligamentous Laxity
}

\section{Madison Goss*, Grace 0'Neill and William Hennrikus}

Department of Orthopaedics and Rehabilitation, Penn State College of Medicine, United States of America

*Corresponding Author: Madison Goss, Department of Orthopaedics and Rehabilitation, Penn State College of Medicine, Penn State Milton S. Hershey Medical Center, Hershey, PA, United States of America.
Received: March 20, 2020

Published:

(C) All rights are reserved by Madison Goss., et al.

\begin{abstract}
Objective: To report the epidemiology, risk factors and treatment outcome for Nursemaid's elbow in children. The current literature reports recurrence rates for Nursemaid's elbow to be between 14 - 39\%, with some reports of a higher prevalence of ligamentous laxity in children with this injury compared to those without.

Study Design: A retrospective chart review was done at a Children's Hospital for 24 pediatric patients who presented acutely with Nursemaid's elbow. The patients' charts were reviewed for epidemiology, evidence of ligamentous laxity, mechanism of injury, side of injury, reduction techniques, and recurrence.

Results: The study population had an average age 2.4 years old with a female predominance (66\%). Of the left sided injuries, the most common mechanism of injury was a pulled elbow (71\%) stemming from axial traction and pronation of the left forearm. The reduction maneuvers used were the supination/flexion (73\%) and pronation/extension (27\%). Recurrence of Nursemaid's elbow was demonstrated in $71 \%$ of patients. All patients demonstrated ligamentous laxity.

Conclusion: The most common mechanism of injury for Nursemaid's elbow was an elbow pull injury causing the annular ligament to partially get caught in the radio-capitellar joint. All patients demonstrated ligamentous laxity. The most common reduction method was supination/flexion. Recurrence was high at $87 \%$ in males and $63 \%$ in females. Left sided injuries were predominantly caused by an elbow pull, likely due to a right-handed adult holding the child's left hand at the time of injury. No predominant mechanism was observed for right-sided injuries.

Keywords: Annular Ligament Subluxation; Retrospective Study; Mechanism of Injury; Joint Hypermobility
\end{abstract}

\section{Introduction}

Annular ligament subluxation, also known as "Nursemaid's Elbow," is a common injury of early childhood most often occurring in children ages 1 - 4 with a peak incidence at age 2 - 3 [1]. Nursemaid's elbow occurs when the annular ligament subluxates on the radial head, most often as a result of axial traction applied to the child's forearm that occurs when an adult pulls a child by the wrist (Figure 1). Generalized ligamentous laxity has been suggested as a risk factor for musculoskeletal injuries in Nursemaid's elbow [2]. Children who sustain this injury typically have ligamentous laxity. This laxity allows the annular ligament to subluxate out of its anatomic position around the radial neck to the radial head and become trapped in the radio-capitellar joint.

This injury is usually able to be treated with reduction maneuvers that may be performed in emergency departments or primary care offices without the need for sedation or intervention by an orthopaedic surgeon. Reduction maneuvers include the hyperpronation maneuver and the supination-flexion maneuver. One prior analysis has shown hyperpronation to be a superior treatment for Nursemaid's elbow when compared to supination-flexion in terms of success rate and possibly less procedural pain [3]. The purpose

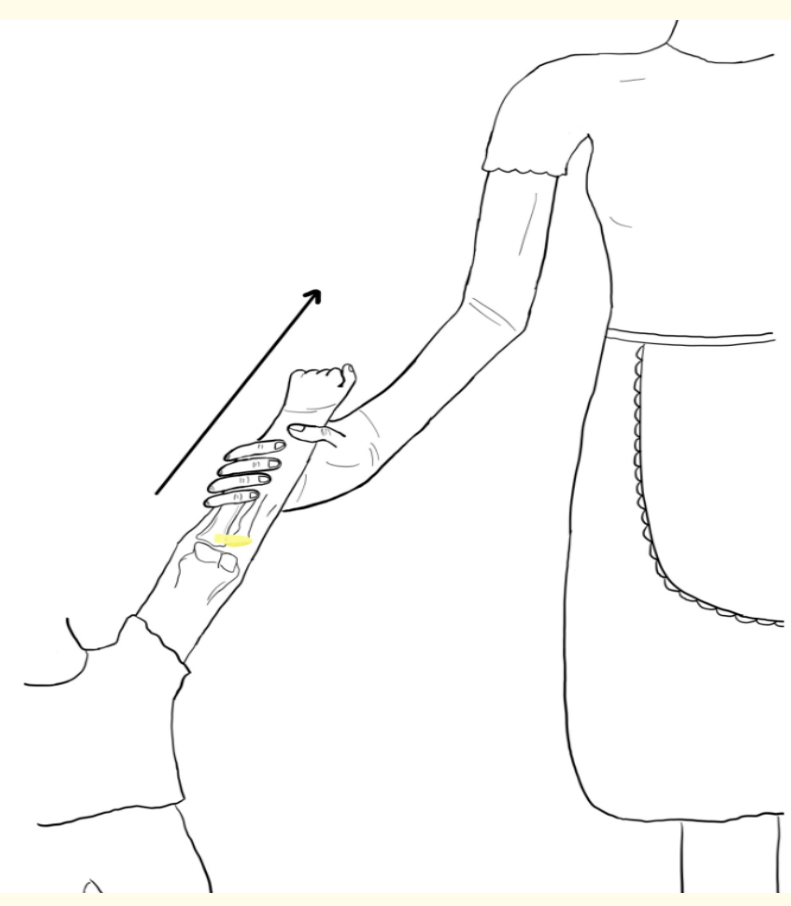

Figure 1: Most common mechanism of injury in nursemaid's elbow. The classic mechanism of injury for which nursemaid's elbow was named occurs when a child's arm is pulled by a taller adult allowing annular subluxation to occur. 
of this study was to investigate the following three objectives in patients with a diagnosis of Nursemaid's elbow at a single institution:

1. Describe the epidemiology

2. Identify risk factors

3. Report the outcomes of treatment for Nursemaid's elbow in children.

\section{Materials and Methods}

Approval from the institutional review board was obtained prior to data collection. A retrospective chart review of billing records was performed for patients whose charts noted a diagnosis of Nursemaid's elbow at our institution during 2016. Inclusion criteria consisted of age between neonate and 15 years of age at the time of injury and documented follow up within 14 days of the initial injury. For each patient that met inclusion criteria, the following characteristics were noted: age, gender, weight with percentile for age and gender, laterality, mechanism of injury, use of radiographs, reduction method/treatment techniques, use of sedation, and recurrence. Descriptive statistics were performed on the data.

\section{Results}

A total of 24 patients met inclusion criteria for this study. The average age was 2.4 years old, ranging from 0.83 to 5 years of age. The average weight percentile of the patients was 65th, with it ranging from 25th to 96th. The percentage of patients with a weight percentile at time of injury greater than 70th was $46 \%$. Nursemaid's elbow was more common in females (66\%). Right and left-sided injuries occurred in near equal frequency (49\%:51\%). The most common mechanism of injury was a pulled elbow injury (52\%) stemming from axial traction and pronation when a parent or a sibling lifted the child by the arm, pulled the child by the arm across the street, or pulled on the arm to place the arm through a shirt sleeve when dressing the child (Figure 2). Of left-sided injuries, a pulling mechanism was most common (Figure 2). No mechanism of injury predominated for right-sided injuries. All patients presented with a tender elbow in pronation and slight flexion. At initial presentation with the primary care physician, radiographs were obtained for only $13 \%$ of patients. The decision for radiographs were made on a case by case basis by the treating physician.

No sedation was used at the time of reduction for any of the patients included. The reduction maneuver most commonly used was the supination/flexion maneuver (73\%) followed by the extension/hyperpronation maneuver (27\%). No casting was used in any of the patients. Recurrence rate for Nursemaid's elbow overall was $71 \%$. In female patients, the recurrence rate was $63 \%$ $(\mathrm{n}=10 / 16)$, while in male patients the recurrence rate was $87 \%$ $(\mathrm{n}=7 / 8)$. All patients had documented ligamentous laxity/joint hypermobility. Ligamentous laxity was determined using several passive range of motion tests including apposition of the thumb to flexor side of forearm, dorsiflexion 90 degrees or greater of the fifth metacarpophalangeal joint, hyperextension of the elbows

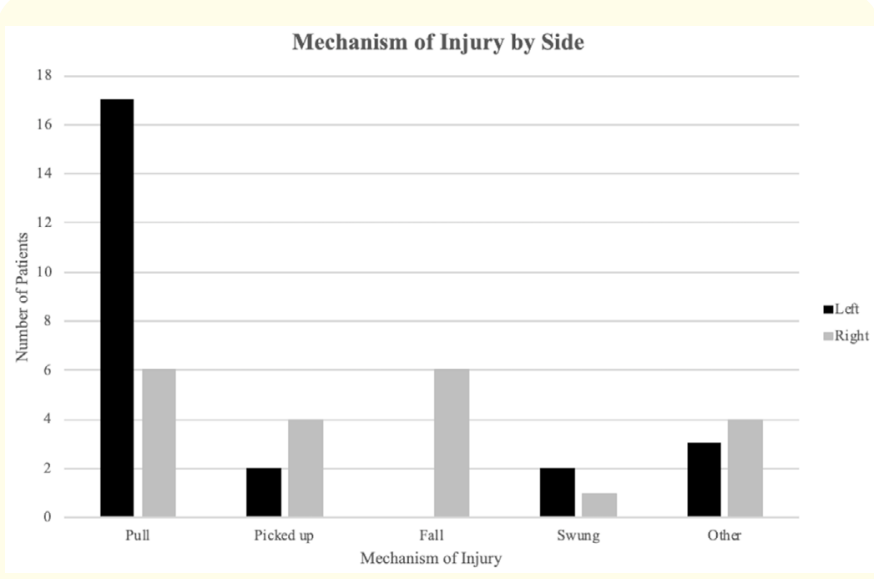

Figure 2: Mechanism by laterality of injury. There was a higher prevalence of left-sided injuries resulting from a pull mechanism when compared to other mechanisms.

and knees 10 degrees or greater, and palms resting flat on the floor with forward flexion of the trunk. These tests are components of the 9-point Beighton score of hypermobility which has shown to be valid for determination of ligamentous laxity in children [4,5].

\section{Discussion}

Consistent with the current literature, we found a higher incidence in female patients (66\%) and in patients with an above-average weight percentile (mean $=65$ th percentile). The most common mechanism of injury documented was a classic pulled elbow injury, for which the nickname Nursemaid's elbow was given. We also found a variety of other mechanisms including falls and one patient that slept on their arm. Where the left arm was injured, a pulling mechanism was the most common mechanism of injury (71\%) (Figure 2). This finding may be explained by higher prevalence of right-handed parents over left-handed parents [6]. Right-handed parents would typically be holding their child's left hand with their own right hand, giving more opportunity for injury of the left arm when compared to the right (Figure 1).

All patients had a documented finding of ligamentous laxity/ joint hypermobility supporting this as a risk factor for radial head subluxation. One case report in 2010 demonstrated an 8 year old girl with Nursemaid's elbow, outside the typical age range of 1 - 4, who was found to have ligamentous laxity on exam along with a family history of joint hypermobility [7]. It has been demonstrated in the literature that there is a higher prevalence of ligamentous laxity in children who have sustained a Nursemaid's elbow injury when compared to their peers who have not [8]. Interestingly, the parents of children who sustained a Nursemaid's elbow injury also have a higher prevalence of ligamentous laxity suggesting a role for genetics in the risk of acquiring a Nursemaid's elbow injury [8].

In the current study, the most commonly used reduction technique was supination/flexion. The maneuver with the lowest rate of 
recurrence at $74 \%$ was also supination/flexion. Other maneuvers such as pronation/flexion had recurrence rates of $100 \%$, but this may be due to the small sample size for these less commonly used maneuvers at our institution. The most commonly used reduction method for different institutions is often dependent upon the preference and training of the teaching faculty. Previously reported recurrence rates of nursemaid's elbow have been variable, anywhere from 14\% - 39\% [9]. This inconsistency has been attributed to varied methods of data collection including relying on recall of previous similar episodes from caregivers, versus following the patients over time to watch for recurrence [10]. Overall, the recurrence rate in our population was high at $71 \%$ for children with at least one recurrence. Some children had more than one recurrence, with one patient having 4 recurrences in addition to the initial injury. Identifying children at a higher risk for recurrence at initial presentation could be used to provide parents with additional education regarding prevention of recurrence by minimizing the activities that lead to injury which could potentially decrease the number of emergency room visits and unnecessary radiographs obtained. Overall, the high recurrence rate is underreported in primary care literature.

The key findings of this study are:

1. Female predominance was seen in this patient population

2. Ligamentous laxity was observed in all patients

3. Recurrence of Nursemaid's elbow is common

4. Either reduction technique works in the treatment of Nursemaid's elbow.

The most common mechanism of injury for Nursemaid's elbow was an elbow pull injury causing the annular ligament to partially get caught in the radio-capitellar joint. The most common reduction method was supination/flexion in which the physician supinates the hand, extends the arm, then flexes the elbow. Due to ligamentous laxity and re-injury, recurrence was high at $87 \%$ in males and $63 \%$ in females. The left side preponderance is most likely due to the more prevalent right-handed adult holding the child's left hand at the time of the elbow pull injury.

\section{Conclusion}

In conclusion:

1. The epidemiology of Nursemaid's elbow includes the predominance of female patients and left-sided injuries due to a pulling mechanism.

2. Ligamentous laxity is the \#1 risk factor for Nursemaid's elbow injury.

3. Outcomes are routinely good, however $>70 \%$ of patients at least 1 recurrence of Nursemaid's elbow.

\section{Conflict of Interest}

No conflicts of interest exist for this manuscript.

\section{Bibliography}

1. Irie T., et al. "Investigation on 2331 Cases of Pulled Elbow over the Last 10 Years". Pediatric Reports 6.2 (2014): 5090.
2. Bin Abd Razak HR., et al. "Generalized Ligamentous Laxity May Be a Predisposing Factor for Musculoskeletal Injuries". Journal of Science and Medicine in Sport 17.5 (2014): 474-478.

3. Bexkens R., et al. "Effectiveness of Reduction Maneuvers in the Treatment of Nursemaid's Elbow: A Systematic Review and Meta-Analysis". American Journal of Emergency Medicine 35.1 (2017): 159-163.

4. Bird HA., et al. "Quantification of Joint Laxity". Rheumatology and Rehabilitation 18.3 (1979): 161-166.

5. Smits-Engelsman B., et al. "Beighton Score: A Valid Measure for Generalized Hypermobility in Children". Journal of Pediatrics 158.1 (2011): 119-123.

6. Michel GF., et al. "Evolution and Development of Handedness: An Evo-Devo Approach". Progress in Brain Research 238 (2018): 347-374.

7. Barton MA. "Radial Head Subluxation in an 8-Year-Old Girl with Joint Hypermobility". BMJ Case Reports (2010).

8. Amir D., et al. "Pulled Elbow and Hypermobility of Joints". Clinical Orthopaedics and Related Research 257 (1990): 94-99.

9. Teach SJ and SA Schutzman. "Prospective Study of Recurrent Radial Head Subluxation". Archives of Pediatrics and Adolescent Medicine 150.2 (1996): 164-166.

10. Wong K., et al. "Radial Head Subluxation: Factors Associated with Its Recurrence and Radiographic Evaluation in a Tertiary Pediatric Emergency Department". Journal of Emergency Medicine 51.6 (2016): 621-627.

\section{Assets from publication with us}

- Prompt Acknowledgement after receiving the article

- Thorough Double blinded peer review

- Rapid Publication

- Issue of Publication Certificate

- High visibility of your Published work

Website: https://www.actascientific.com/

Submit Article: https://www.actascientific.com/submission.php Email us: editor@actascientific.com

Contact us: +919182824667 